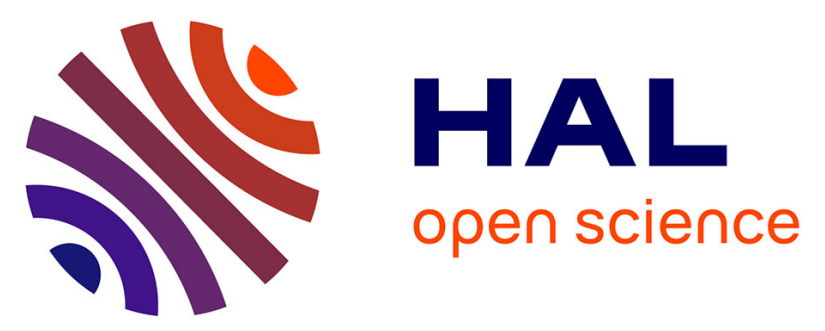

\title{
Experimental data analysis on xenon above the critical temperature from nonlinear renormalization group
}

\author{
C. Bagnuls, C. Bervillier, Y. Garrabos
}

\section{To cite this version:}

C. Bagnuls, C. Bervillier, Y. Garrabos. Experimental data analysis on xenon above the critical temperature from nonlinear renormalization group. Journal de Physique Lettres, 1984, 45 (3), pp.127132. 10.1051/jphyslet:01984004503012700 . jpa-00232318

\section{HAL Id: jpa-00232318 https://hal.science/jpa-00232318}

Submitted on 1 Jan 1984

HAL is a multi-disciplinary open access archive for the deposit and dissemination of scientific research documents, whether they are published or not. The documents may come from teaching and research institutions in France or abroad, or from public or private research centers.
L'archive ouverte pluridisciplinaire HAL, est destinée au dépôt et à la diffusion de documents scientifiques de niveau recherche, publiés ou non, émanant des établissements d'enseignement et de recherche français ou étrangers, des laboratoires publics ou privés. 
Classification

Physics Abstracts

$64.70 \mathrm{~F}-64.70$

\title{
Experimental data analysis on xenon above the critical temperature from nonlinear renormalization group
}

\author{
C. Bagnuls
}

Service de Physique du Solide et de Résonance Magnétique, CEN-Saclay, 91191 Gif sur Yvette Cedex, France

C. Bervillier

Service de Physique Théorique, CEN-Saclay, 91191 Gif sur Yvette Cedex, France

and Y. Garrabos

Laboratoire des Interactions Moléculaires et des Hautes Pressions, CNRS, Centre Universitaire Paris-Nord, avenue J. B. Clément, 93430 Villetaneuse, France

(Reçu le 9 novembre 1983, accepté le 13 décembre 1983)

\begin{abstract}
Résumé. - Les données expérimentales, pour le xénon, de la lumière diffusée et de la chaleur spécifique sont analysées à partir des calculs récents obtenus pour le modèle $\phi^{4}$ à trois dimensions. L'ensemble réduit des paramètres libres que la théorie impose est suffisant pour décrire ces données même lorsque, dans l'analyse habituelle, de nombreux termes de correction aux lois d'échelle sont nécessaires. Nous montrons qu'il sera très difficile de déterminer l'influence des termes transitoires négligés dans le modèle $\phi^{4}$. De plus, nous obtenons la réduction à des quantités expérimentales sans dimension en accord avec l'universalité à deux échelles près. Nous montrons que les amplitudes asymptotiques des quantités singulières sont aussi reliées à celles des premières corrections confluentes, selon le chemin critique considéré.
\end{abstract}

\begin{abstract}
We analyse the scattered light and specific heat data on xenon using the recent nonlinear renormalization group calculations obtained in the $\phi^{4}$ theory in three dimensions. The minimal set of free parameters that the theory imposes is sufficient to describe these data even for the case where, in the usual analysis, many correction terms to scaling are needed. We show that it will be very difficult to determine the influence of transients neglected in the $\phi^{4}$ model. Furthermore, we obtain the reduction to dimensionless experimental quantities in agreement with two scale factor universality. We show that the asymptotic amplitudes of the singular quantities are also related to the amplitudes of the first confluent corrections according to the critical path considered.
\end{abstract}

\section{Introduction.}

Recently, confluent correction terms to scaling have been shown to be necessary in the analysis of experimental data on xenon [1] at equilibrium along its critical isochore above $T_{\mathrm{c}}$ (the critical temperature). These corrections should be needed especially in the analysis of data on fluids in which the approach to the critical point is limited (e.g. gravity effects [2]). In the case where many correction terms are needed, the adjustable parameters become too numerous to be well determined. This is correlated to the slow convergence of the Wegner expansion [3]. 
In the preceding letter [4], a new kind of comparison between experiment and renormalization group (RG) theory is proposed. It is based upon results obtained from a nonlinear analysis of the $\phi^{4}$ Landau-Ginzburg-Wilson model in three dimensions $(d=3)$. The complete set (susceptibility $\chi$, correlation length $\xi$ and specific heat $C$ ) of measurable quantities along the critical isochore above $T_{\mathrm{c}}$, has been obtained as explicit functions of the dimensionless scaling field $t^{*}$ which vanishes with $\tau=\left(T-T_{\mathrm{c}}\right) / T_{\mathrm{c}}$. It is shown that only four adjustable parameters should be introduced to reproduce the real behaviours of $\xi, \chi$ and $C$, even in the nonasymptotic critical region where corrections are relevant. Two among these four parameters $(\theta$ and $\psi)$ represent the two nonuniversal scales related to $\tau$ and $H$ (the ordering field conjugated to the order parameter) which characterize a given system within a universality class. A third one $\left(g_{0}\right)$ is related to the process of reduction to dimensionless quantities for allowing comparison between experiments and field theory calculations. The fourth is not a critical parameter : it is the regular part of $C$ (the true background term) which should not be confused with its critical additive part.

This letter is devoted to an illustration, on xenon, of the use of the nonasymptotic theoretical calculations given in table I of [4]. The $\phi^{4}$ model, used to do the calculations, does not take into account all the characteristics of real systems as one goes far from $T_{\mathrm{c}}$. Hence, deviations between the theoretical functions and the experimental data should occur from some value $\tau_{\mathrm{d}}$ as one goes away from $T_{\mathrm{c}}$.

The comparison with experimental data is given in section 2 . In section 3 we discuss the reduction process to dimensionless quantities which for the sake of clarity is not considered in section 2 .

\section{Analysis of xenon data.}

To allow a complete illustration of the use of the nonasymptotic functions of [4], we need independent measurements concerning $\xi, \chi$ and $C$ along the critical isochore above $T_{\mathrm{c}}$. Recently, Güttinger and Cannell [1] have measured very precisely the intensity of scattered light and the turbidity $\mathfrak{C}$ in xenon. These two kinds of measurements give us the opportunity to compare them with our theoretical $\xi^{*}$ and $\chi^{*}$. To be complete, and to illustrate two scale factor universality [5]. we also need measurements of the specific heat. We have used the data of Edwards et al. [6] although they do not present the same level of accuracy, what is not important for our illustration.

The data will be analysed be using test functions referred to by the subscript " exp », related to our dimensionless (starred) theoretical functions given in table I of reference [4] as follows :

$$
\begin{aligned}
& \chi_{\exp }(\tau)=\left(\Gamma^{+}\right) \chi^{*}(\theta \tau) \\
& \xi_{\text {exp }}(\tau)=\left(\xi_{0}^{+}\right) \xi^{*}(\theta \tau) \\
& C_{\text {exp }}(\tau)=\left(A^{+} / \alpha\right) C_{\mathrm{s}}^{*}(\theta \tau)+B_{\text {exp }} .
\end{aligned}
$$

The functions $\chi^{*}(x), \xi^{*}(x)$ and $C^{*}(x)$ are defined for any value of $x$ in the range $0 \lesssim x \lesssim \infty$ [4]. $C_{\mathrm{s}}^{*}(x)$ is the singular part of the function $C^{*}(x)\left(C_{\mathrm{s}}^{*}(x)=C^{*}(x)-B, B\right.$ is also given in table $\mathrm{I}$ of [4]).

The parameters $\Gamma^{+}, \xi_{0}^{+}, A^{+}, B_{\exp }$ and $\theta$ entering into the equations (1) differ from the nonuniversal $\left(\theta, \psi, g_{0}\right.$ and $\left.C_{\text {reg }}\right)$ pardmeters discussed in [4]. Indeed, we have introduced another way to write them down to account for the nonuniversality (related to $g_{0}$ in [4]) involved in the reduction process to dimensionless experimental quantities (see next section). $B_{\text {exp }}$ combines the regular part $C_{\text {reg }}$ of $C$ (the background) with the critical additive constant $B$. We have no more than four adjustable parameters if we note that $A^{+}$is related to $\xi_{0}^{+}$by two scale factor universality through [5] :

$$
R_{\xi}^{+}=\xi_{0}^{+} \times\left(A^{+}\right)^{1 / 3} .
$$

Let us note that the scale $\theta$ so introduced in equations (1) is closely related to the existence of corrections to scaling. The nonuniform experimental accuracy will, unfortunately, prevent us 
from obtaining a globally satisfactory determination of all the parameters within each function (especially for $\theta$ ). Our aim is to outline the general principles of the comparison which allows us to determine the range of $\tau$ where the $\phi^{4}$ model is valid and the critical domain reached. The spirit of the determination of the adjustable parameters will thus not be able to seek the best fit of the data but to look for a global agreement within an a priori supposed experimental accuracy.

Let us now deal with the determination of the adjustable parameters from the experimental data.

In principle, the light scattering data have to be analysed using the susceptibility and the correlation length together within the correlation function in its Ornstein-Zernicke $(\mathrm{OZ})$ approximation. However, the particular presentation in [1] of the susceptibility data to a reference temperature $\tau_{\mathrm{r}}$ provides the opportunity of analysing them with only $\theta$ as an adjustable parameter by using $\chi^{*}(\theta \tau) / \chi^{*}\left(\theta \tau_{\mathrm{r}}\right)$ as the test function (see Eq. (1a)). We have also considered $T_{\mathrm{c}}$ an adjustable parameter as in [1]. No systematic deviations have been observed between our test function and the data in the range of values of $\tau$ considered $\left(10^{-4} \lesssim \tau \lesssim 10^{-1}\right)$ with $\theta=(1.91 \pm 0.85) \cdot 10^{-2}$. The estimated error on $\theta$ corresponds to a supposed experimental accuracy of $1 \%$. Let us indicate, however, that the largest distance between the experimental points and the test function, for $\theta=1.91 \times 10^{-2}$, was less than $0.4 \%$ and that no significative variation of $T_{\mathrm{c}}$ was observed as was also the case in [1]. The fact that we obtain a value for $\theta$ corroborates the existence of the corrections seen by Güttinger and Cannell.

We next analysed the turbidity data $\mathcal{G}$ which mix $\chi$ and $\xi$ within the correlation function (considered in the $\mathrm{OZ}$ approximation), according to [7] :

where :

$$
\mathcal{C}=\pi \mathfrak{A} \chi_{\exp }(\tau) \times f(z(\tau))
$$

$$
f(z)=\frac{2 z^{2}+2 z+1}{z^{3}} \ln (1+2 z)-\frac{2(1+z)}{z^{2}}
$$

and :

$$
z(\tau)=2\left(k_{0} \xi_{\exp }(\tau)\right)^{2}
$$

where $k_{0}$ is the wave vector of the light in the fluid and $\mathcal{A}$ is a known constant [7]. We have three adjustable parameters : $\xi_{0}^{+}, \Gamma^{+}$and $\theta$. As the relative experimental errors in turbidity grow with $\tau$. $\theta$ is not really well defined by the turbidity measurements, unlike $\Gamma^{+}$and $\xi_{0}^{+} . \theta$ being fixed at $1.91 \times 10^{-2}$, we have then found :

$$
\begin{aligned}
\left(\Gamma^{+}\right)^{*} & =\frac{P_{\mathrm{c}}}{\zeta_{\mathrm{c}}} \Gamma^{+}=0.057 \pm 0.01 \\
\xi_{0}^{+} & =(1.83 \pm 0.02) \AA
\end{aligned}
$$

When $\theta$ was left free, we found $\theta=(2 \pm 3) \cdot 10^{-2},\left(\Gamma^{+}\right)^{*}=0.057 \pm 0.01$ and $\xi_{0}^{+}=(1.87 \pm$ $0.03) \AA$. The errors correspond to a supposed (constant) absolute experimental error of $10^{-3}$ on the turbidity. Within this accuracy, no systematic deviation has been observed in the range of $\tau$ considered. These values must be compared with those of [1] which are : $\Gamma^{+}=0.0577 \pm 0.001, \xi_{0}^{+}=1.84 \AA$, while the universal ratio of the first correction amplitudes $\left(a_{\xi}^{1} / a_{\chi}^{1}\right.$, see relation (3)) was not found to be in agreement with the RG prediction $(0.42$ instead of $0.64[8]$ ).

For the specific heat data, we have taken into account gravity effects as did Edwards et al. [6] by using the above determination of the susceptibility. The adjustable parameters are $A^{+}, \theta$ and $B_{\text {exp. }}$. However, the measurements of $C$ are not so accurate as those of the scattered light. It has thus not been possible to obtain a good determination of $\theta$. We simply indicate that the asymptotic amplitude $A^{+}$is compatible with the value $\xi_{0}^{+}=(1.86 \pm 0.01) \AA$ (by using Eq. (2) with $\left.R_{\xi}^{+} \sim 0.27[8]\right)$ and $B_{\exp }=(-57.2 \pm 4) \mathrm{J} /$ mole K. No systematic deviation has been 
observed within a supposed experimental error of $2 \%$ while $\theta$ is not determined. This latter point illustrates the fact that such data were fitted without correction to scaling in [6].

We can conclude from this comparison that the asymptotic critical behaviour predicted by RG theory is in a good agreement with experiments. Furthermore, the most precise measurements (light scattering) indicate that, for xenon, the value $\tau_{d}$ at which deviations between the $\phi^{4}$ model and the real system appear is greater than $10^{-1}$.

It is possible to point out that such a $\tau_{\mathrm{d}}$ exists by looking at the variation of the effective exponent $\gamma_{\text {eff }}=-(\mathrm{d} \ln (\chi) / \mathrm{d} \ln (\tau))$ as a function of $\tau$ and comparing the $\phi^{4}$ model (with the parameter $\theta$ adjusted to the value $\left(1.91 \times 10^{-2}\right)$ determined above) to a rough estimate of $\gamma_{\text {eff }}$ from various experiments over a large range of $\tau$ (Fig. 1). We can say that owing to the large value of $\tau_{\mathrm{d}}$ it will not be possible to reduce these deviations without considering a nonlinear study of the transients not considered in the $\phi^{4}$ model, unless the experimental errors can be reduced.

We shall now discuss partially the problem of the reduction to dimensionless experimental quantities (the reduction process).

\section{Correspondence between the RG calculations and the real fluids.}

To set up clearly the question that we discuss in this section let us expand the equations $(1(b)$ and $(c))$ up to the first correction and reexpress the asymptotic amplitudes $A^{+}, \Gamma^{+}$and $\xi_{0}^{+}$as they come from the theory [4] :

$$
\begin{aligned}
& \xi_{\text {exp }}(\tau)=\left(Z_{\xi} / g_{0}\right)(\theta \tau)^{-v}\left(1+a_{\xi}^{1}(\theta \tau)^{\Delta}+\cdots\right) \\
& C_{\text {exp }}(\tau)=\theta^{2}\left\{Z_{\mathrm{c}} g_{0}^{3} \times(\theta \tau)^{-\alpha}\left(1+a_{\mathrm{c}}^{1}(\theta \tau)^{\Delta}+\cdots\right)+B\right\}+C_{\text {reg }} .
\end{aligned}
$$

In these expressions, $Z_{\xi}, Z_{\mathrm{c}}, B, a_{\xi}^{1}$, and $a_{\mathrm{c}}^{1}$ are numerically known from table I of [4] (see footnote [8]). $\theta, g_{0}$ and $C_{\text {reg }}$ are the adjustable parameters. As already said $\theta$ characterizes the temperature scale of a given system while $g_{0}$ (an inverse length in the theory), which comes from dimensional analysis, is also characteristic of the system. The two scale factor universality which states that only two scales (related to $\tau$ and $H$ ) characterize a real system in a given class of universality is well illustrated here if one considers the nonuniversal scales as being $\theta$ and $\psi(\psi$ should appear in Eq. $(1 a)$ in $\left.\Gamma^{+}\right)$. As a consequence not only is the combination $R_{\xi}^{+}=\xi_{0}^{+}\left(A^{+}\right)^{1 / 3}=Z_{\xi}\left(\alpha Z_{\mathrm{c}}\right)^{1 / 3}$ independent of $\theta$ (and $g_{0}$ ) and thus universal but the ratio $a_{\xi}^{1} / a_{\chi}^{1}$ is also. We note that the dependence on $g_{0}$ disappears in $R_{\xi}^{+}$because it is a dimensionless quantity. Apart from this point the universality of $R_{\xi}^{+}$and of $a_{\xi}^{1} / a_{\chi}^{1}$ comes from the unique scale which caracterizes the critical isochore. From this it will be interesting to look for a reduction process to dimensionless experimental quantities which could give an estimation of $\theta$ from the asymptotic amplitude alone, even if the corrections to scaling are not observed from data analysis.

In the following we shall essentially concentrate the discussion on the relation between $g_{0}$ and real systems. Only the critical isochore is considered and the case of the critical isotherm (related to $\psi$ ) is discussed elsewhere [9].

Let us first recall the respective position of the three independent nonuniversal parameters $g_{0}, \theta$ and $\psi$ :

(i) $g_{0}$ characterizes the critical point of the fluid considered and depends only on the coordinates of the critical point.

(ii) $\theta$ is a parameter which characterizes the critical isochore of the fluid.

(iii) $\psi$ is the scale characterizing the critical isotherm of the fluid.

(iv) Once $g_{0}, \theta$ and $\psi$ are determined for a particular fluid, the critical behaviour is, in principle, completely known from the calculations of the amplitudes $Z$ and $a^{1}$ in equations $(3,4)$. These amplitudes are the same for all fluids. 


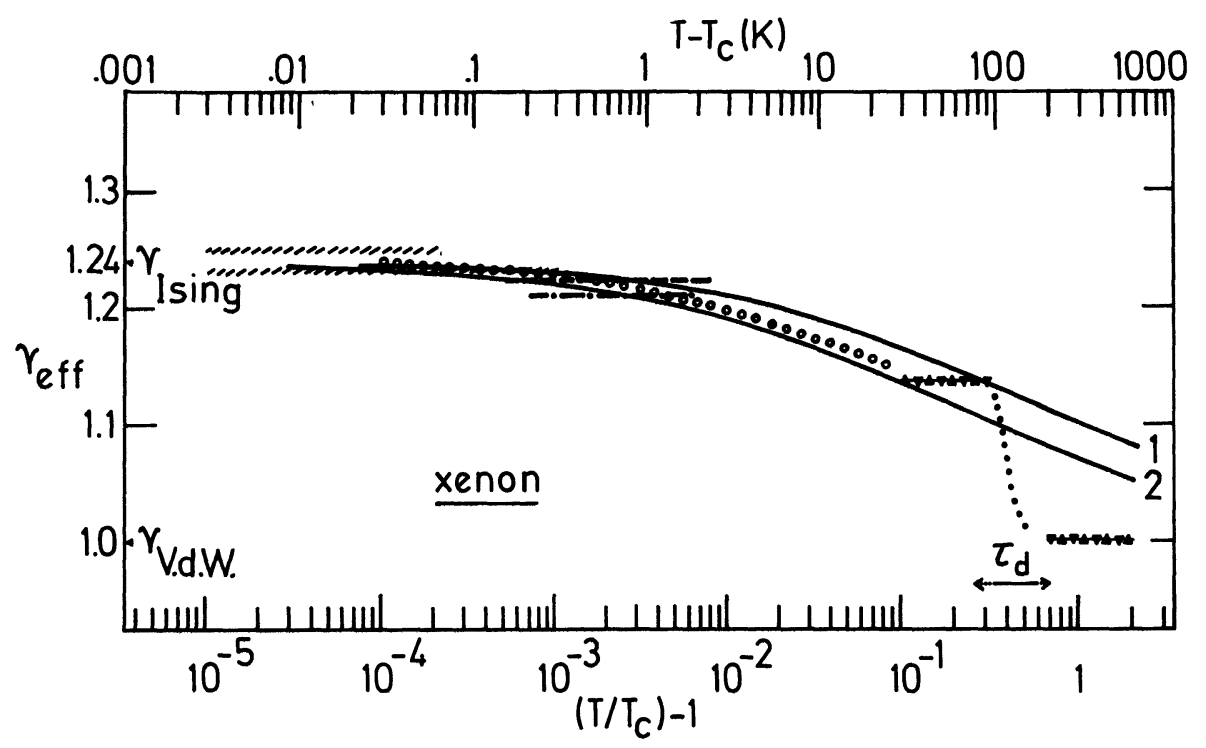

Fig. 1. - Variations of the effective exponent $\gamma_{\text {eff }}$ - defined in the text - versus the distance $\tau$ to the critical temperature. The full curves (1 and 2) represent the theoretical variations of $\gamma_{\text {eff }}$ for $\theta=2.8 \times 10^{-2}$ and $\theta=1 \times 10^{-2}$ respectively. The other symbols refer to experimental estimations : $/ / / /$ from interferometric profile data (W. T. Estler, R. Hocken, T. Charlton and L. R. Wilcox, Phys. Rev. A 12 (1975) 2118). ---- from light scattering data (see ref. [9]). 0000 from light scattering data (D. S. Cannell and H. Güttinger, Proceedings of the Eighth Symposium on Thermophysical Properties, Ed. J. V. Sengers (1981), and ref. [1]). - - - - - from PVT data (J. M. H. Levelt-Sengers, W. L. Greer and J. V. Sengers, J. Phys. Chem. 5(1976) 1). $\Delta-\nabla-\Delta$ from PVT data (see ref. [9]). The data which were fitted by a pure scaling law in the whole range of $\tau$ considered give a constant $\gamma_{\text {eff }}$. The PVT data clearly show that $\phi^{4}$ calculations do not describe the real fluid for large $\tau\left(\tau_{\mathrm{d}} \sim 5 \times 10^{-1}\right)$.

We shall now show that $g_{0}^{-1}$ is related to a length characteristic of the fluid through a universal relation between the coordinates of the critical point such that only two nonuniversal scales $(\theta$ and $\psi)$ characterize the fluid.

Since $g_{0}$ is defined at the critical point, it depends only on $P_{\mathrm{c}}, \zeta_{\mathrm{c}}$ and $T_{\mathrm{c}}$ (the critical pressure, density and temperature of the fluid) and the relation $g_{0}^{-1}\left(P_{\mathrm{c}}, m / \zeta_{\mathrm{c}}, T_{\mathrm{c}}\right)$ is the same for all fluids ( $m$ is the molecular mass of the fluid). Since $P_{c}, m / \zeta_{c}$ and $T_{c}$ are related through the equation of state only two independent variables enter into $g_{0}^{-1}$. Thus from dimensional argument $g_{0}^{-1}$ reads :

$$
g_{0}^{-1}=l_{l}^{*}\left(z_{\mathrm{c}}\right)
$$

where $l$ is a length which can be chosen as :

$$
l=\left(k_{\mathrm{B}} T_{\mathrm{c}} / P_{\mathrm{c}}\right)^{1 / 3}
$$

in that case $z_{\mathrm{c}}=\left(P_{\mathrm{c}} /\left(k_{\mathrm{B}} T_{\mathrm{c}}\right)\right) \times m / \zeta_{\mathrm{c}}$. For that particular choice of $l, f_{l}{ }^{*}$ is the same for all fluids. From this and equations $(1(b), 3)$ we obtain the following relation between the amplitude $\xi_{0}^{+}$ and the theory :

$$
\xi_{0}^{+} / l=Z_{\xi} \times(\theta)^{-v} \times f_{l}^{*}\left(z_{\mathrm{c}}\right)
$$


The value of $\theta$ obtained in section 2 yields [10] :

$$
2.5 \times 10^{-2} \lesssim f_{l}^{*}\left(z_{\mathrm{c}}^{\mathrm{xenon}}\right) \lesssim 4.65 \times 10^{-2} .
$$

This estimation, for xenon, does not give the « universal » function $f_{l}{ }^{*}$. However any fluid for which $z_{\mathrm{c}}=z_{\mathrm{c}}^{\mathrm{xen}}$ (rare gases) will be characterized by the same value of $g_{0}^{-1}=(0.32 \pm 0.1) \AA$, provided that the choice of $l$ is that of (6).

The same considerations could be used on the singular part of the specific heat but do not give more information on $f_{l}^{*}$ owing to the universal relation $R_{\xi}^{+}$.

The relation between field theory calculations and experiments is not limited to $\xi$ and $C$ and could be extended to $\chi$ which requires to consider the scale $\psi$. Since the theoretical calculations along the critical isotherm have not been yet achieved we shall not pursue this discussion here. Further general consequences of the particular form predicted by the RG theory are presented elsewhere by Y. Garrabos [9].

In summary, we have shown, for xenon, how to use the calculations from the $\phi^{4}$ model of [4] coherently with the principles of the $R G$ approach. The number of adjustable parameters are considerably reduced and will lead to improved comparisons with experiments, especially to a better characterization of the corrections to scaling.

By relating the coupling constant $g_{0}$ to the reduction process, we have illustrated the fact that the leading and confluent amplitudes of all the singular quantities along any critical path are related. The knowledge of only two scales $(\theta$ and $\psi)$ determines all the critical behaviours up to the first Wegner correction.

We hope that further comparisons with experiments will be made in other systems.

\section{References}

[1] GÜttinger, H. and CANNell, D. S., Phys. Rev. A 24 (1981) 3188.

[2] Hohenberg, P. C. and Barmatz, M., Phys. Rev. A 6 (1972) 289.

[3] Wegner, F. J., Phys. Rev. B 5 (1972) 4529.

[4] Bagnuls, C. and Bervillier, C., J. Physique Lett., this issue.

[5] Stauffer, D., Ferer, M. and Wortis, M., Phys. Rev. Lett. 29 (1972) 345.

[6] Edwards, C., Lipa, J. A. and Buckingham, M. J., Phys. Rev. Lett. 20 (1968) 496 ; Phys. Rev. A 15 (1977) 778 and AIP document no PAPS PLRAA-15-778-15.

[7] Puglielli, V. G. and Ford, N. C., Phys. Rev. Lett. 25 (1970) 143.

[8] To do this comparison we use only the theoretical upper bound of the calculations presented in [4] which corresponds to $v=0.6305, \gamma=1.24194, \alpha=0.1085, \Delta=0.491, Z_{\xi}=0.47, Z_{\chi}=0.263$, $Z_{\mathrm{c}}=1.734, a_{\xi}^{1}=5.33, a_{\chi}^{1}=8.27, a_{\mathrm{c}}^{1}=8.12$.

[9] Garrabos, Y., Thesis, University of Paris, 1982, and in preparation.

[10] For xenon : $P_{\mathrm{c}}=5.84 \times 10^{6} \mathrm{~Pa}, \zeta_{\mathrm{c}}=1110 \mathrm{~kg} / \mathrm{m}^{3}, T_{\mathrm{c}}=289.72 \mathrm{~K}, m=0.1313 \mathrm{~kg}$. 